\title{
Film-Controlled Synchronized Release Multicomponent Preparations of Chinese Materia Medica for Oral Administration
}

\author{
Xin Huang ${ }^{1}$, Yuqin Wen ${ }^{1}$, Qingchun Xie ${ }^{1}$, Lou Shen ${ }^{1}$, Bohong Guo ${ }^{1}$, Chaofeng Liang ${ }^{1,2}$, Yanzhong Chen ${ }^{1 *}$, \\ and Zhufen $\mathrm{Lu}^{1^{*}}$ \\ ${ }^{1}$ Guangdong Provincial Key Laboratory of Advanced Drug Delivery Systems, Guangdong Pharmaceutical University, Guangzhou, China \\ ${ }^{2}$ Guangzhou Pharmaceuticals Corporation, Guangzhou, China
}

e-mail: doctor.c@163.com luzhufen@163.com

\begin{abstract}
Traditional Chinese medicine is mostly compound medicine, and its efficacy is achieved based on the action of multiple components and targets. The simultaneous release of multiple components is the focus of the modernization of traditional Chinese medicine. In view of this, membrane-controlled-release technology can delay the release of pills after a certain time for presenting the characteristics of synchronous release. In this study, a traditional Chinese medicine compound known as "shuxiong" was selected as the research model. The coating formulation was optimized using a central composite design (CCD)-response surface method, and the cumulative release rates of notoginsenoside $R_{1}$, ginsenoside $R b_{1}$, ginsenoside $\mathrm{Rg}_{1}$, and other active components were predicted using the model. Croscarmellose sodium (CCMC-Na) and ethyl cellulose aqueous dispersions were used as the swelling and controlled-release layer materials, respectively. The time lag of the Chinese medicine was extended to $4-5 \mathrm{~h}$, and the cumulative drug release rate was greater than $80 \%$ when the swelling coating and controlled-release coating weights were increased by $15 \%$ and up to $7 \%$, respectively. In this study, the membrane-controlled-release technique allowed for simultaneous release of shuxiong and provided a reference for further development of simultaneous release of compound Chinese herbal medicines.
\end{abstract}

KEYWORDS: drug delivery system, oral bioavailability, film-controlled, synchronized release, multi-components

\section{INTRODUCTION}

The raditional Chinese medicine is a complex system, which involves the use of formulations composed of multiple components with numerous targets and pleiotropic effects (1-5). The effect of differences in the compatibility of traditional Chinese medicine formulations on their efficacy demonstrates that the compatibility of the components harmonizes and coordinates the additive and antagonistic actions $(6,7)$. The efficacy of traditional Chinese medicine is based on the collective activity of the various components, and therefore, the simultaneous release of various active ingredients. Their synchronous action in the body may mediate the effective onset of traditional Chinese medicines (8-11). The evaluation of traditional Chinese medicine has changed from analyzing one or several main components to the simultaneous determination of the release of multiple components in an attempt to improve oral formulations $(12,13)$. Evaluation of one kind of main component of the traditional medicine is not efficient for multiple drug release. The traditional Chinese medicine recipe known as "shuxiong" is compatible with various other Chinese herbal medicines (14-16). There is no time lag in the transport of the drug components of traditional formulations; hence, it is a prerequisite for the multiple components of traditional preparation to be simultaneously released and absorbed to obtain an overall coordinated effect on the system. However, there are some problems such as large oral doses, inconvenient dosage, and bitter taste $(17,18)$. Drug release systems should reflect the overall concept of simultaneous release of multiple components to obtain a comparably stable curative effect with the improved modern preparation technology and the traditional formulation.

Panax notoginseng saponins (PNS, commonly known as Chinese ginseng, mainly contains notoginsenoside $R_{1}$, ginsenoside $R g_{1}$, and ginsenoside $R b_{1}$ ) are the active fractions isolated from the rhizomes of the Chinese herb $P$. notoginseng, which can increase blood flow, dilate 
blood vessels, reduce blood pressure and myocardial oxygen consumption, and improve immune function and hypoxia tolerance (19-22). Thus, it has been developed into a commonly used drug for cardiovascular diseases in clinical medicine. Studies have shown the degradation of PNS in the strongly acidic environment of the stomach, so the duodenum is the best oral absorption site. Therefore, the enteric preparation of PNS was studied to achieve optimum bioavailability. Compound Chinese medicine preparations only achieve the slow release of multiple components in the gastrointestinal tract, which makes it difficult to meet the requirements for synchronization according to conventional formulation methods. Therefore, the synergistic effects of the formulations are essentially disrupted. The simultaneous release of components with different properties has become a bottleneck of dosage form improvement. In this study, membrane-controlled-release technology was used to delay the release of the active components in traditional Chinese medicine preparations to achieve a slow and synchronous effect on the system.

\section{METHODS AND MATERIALS}

\section{Materials}

PNS was refined using the shuxiong extraction process, and polyethylene glycol 6000 (PEG6000) was purchased from Guangzhou Medical Equipment Co., Ltd (Guangzhou, China). Liquid paraffin was purchased from Tianjin Zhiyuan Chemical Reagent Co., Ltd. (Tianjin, China), and the low-substituted hydroxypropyl cellulose (L-HPC) was purchased from Huzhou Zhanwang Chemical Pharmaceutical Co., Ltd. (Huzhou, China). Aquacoat (surelease) was purchased from Shanghai Colorcon Accessories Co., Ltd., (Shanghai, China) croscarmellose sodium (CCMC-Na) was purchased from FMC Accessories Co., Ltd. (Shanghai, China), and carboxymethylcellulose sodium (CCMS-Na) was purchased from Asahi Kasei Corporation (Shanghai, China). Polyvinylpolypyrrolidone (PVPP) was purchased from the American International Corp. (Shanghai, China), while the PNS reference standards $\left(R_{1}, R g_{1}\right.$, and $\left.R b_{1}\right)$ were purchased from Guangzhou Institute for Drug Control (Guangzhou, China).

\section{Preparation of Drop Pills}

The core pills were prepared using PEG4000 and PEG6000 (2:1) as the matrix, which was placed in a water bath $\left(70-80^{\circ} \mathrm{C}\right)$ until a completely melted dispersion was formed. The liquid was uniformly mixed with the PNS (concentrated liquid density, 1:1) after extraction and purification, and was subsequently stored at $80{ }^{\circ} \mathrm{C}$. The mixture was dropped into a container of liquid paraffin as the condensing agent while the speed and distance of the mechanical device were controlled, and then it was cooled to $5{ }^{\circ} \mathrm{C}$. The liquid paraffin on the surface was removed, and the whole product was sieved to obtain the desired particle size of the core-containing pills.

\section{Coating Process}

A fluidized bed bottom-spray technique was used to coat the drop pills. The operating conditions were as follows: outlet air temperature, $35^{\circ} \mathrm{C}$; preheating time, $5 \mathrm{~min}$; atomized air pressure, $0.16-0.20 \mathrm{MPa}$; peristaltic pump rate, $1.5-2.0 \mathrm{~mL} \times \mathrm{min}^{-1}$; outlet air pressure, $0.3-0.4 \mathrm{MPa}$; temperature, $37.0 \pm 2.0^{\circ} \mathrm{C}$. The pellet cores were fully spread on a fluidized bed area under a fluidized condition for the coating process, then the swelling and controlrelease layer coating solution was sprayed uniformly onto the surface of the core-containing pills. The pellets were subsequently dried on the bed at the same temperature until the coating process was completed, then they were placed in an oven to solidify at $40^{\circ} \mathrm{C}$ for $2 \mathrm{~h}$.

\section{Selection of Swelling Layer Material}

The pellets were coated with the following swelling layer materials separately at a concentration of $2 \%(\mathrm{w} / \mathrm{v})$ : CCMC-Na, CCMS-Na, L-HPC, and PVPP. The coating weight of the swelling and control-release layers was fixed while preparing the coated drop pills to enable the determination of the effect of the various swelling layer materials on the drug release rate of the pills.

\section{Selection of Swelling Coating Layer Thickness}

The effect of timing drug release could be obtained when the swelling layer reached a certain thickness. A coating weight of $8 \%$ surelease solution $(\mathrm{w} / \mathrm{v})$ for the controlrelease layer was fixed at $7 \%$, and $2 \%$ (w/v) CCMC-Na was selected as the swelling layer. The coating weight of the swelling layer was varied at $10 \%, 15 \%, 20 \%, 25 \%$, and $30 \%$ while preparing the coated drop pills. This enabled investigation of the effect of swelling layer thickness on the release rate and time lag of the coated drop pills.

\section{Selection of Controlled-Release Layer Thickness}

When the prescription and dosage of the swelling layer was fixed, the drug could be released at a desired time by controlling the thickness of the controlled-release layer. The coating weight of the $2 \%(\mathrm{w} / \mathrm{v})$ CCMC-Na solution swelling layer was fixed at $15 \%$, while the controlledrelease layer coating weight was varied at $5 \%, 7 \%, 10 \%$, and $15 \%$ while preparing the coated drop pills. This was done to investigate the effect of the controlled-release layer's thickness on the release rate and time lag of the coated drop pills. 


\section{Formula Optimization Using Central Composite Design} (CCD)

Based on the screening of the pellet excipients, the effect of drug release was selected as the evaluation index to optimize the swelling layer coating weight, $X_{1}(10-30 \%)$ and controlled-release layer coating weight, $X_{2}$ (5-15\%). The preliminary study was helpful in choosing the factors for the preparation of favorable prescriptions according to the central composite design (CCD) (Table 1). The levels were determined for each factor, and each combination was a prescription. According to the design table arrangement and the preparation of pills, the release rate at 4,8 , and $12 \mathrm{~h}$ was determined using an in vitro release method. The specific factors and levels of the CCD are shown in Table 1, and the CCD experimental arrangement is shown in Table 2.

Table 1. Factors and Levels of Central Composite Design

\begin{tabular}{|c|c|c|c|c|c|}
\hline \multirow{2}{*}{ Factor } & \multicolumn{5}{|c|}{ Level } \\
\cline { 2 - 6 } & -1.414 & -1 & 0 & 1 & 1.414 \\
\hline $\mathrm{X}_{1} / \%$ & 10 & 12.93 & 20 & 27.07 & 30 \\
\hline $\mathrm{X}_{2} / \%$ & 5 & 6.52 & 10 & 13.48 & 15 \\
\hline
\end{tabular}

Table 2. Central Star Design Experiment Arrangement

\begin{tabular}{|c|c|c|}
\hline Run & Factor $1 \mathrm{~A}: \mathrm{X}_{1}$ & Factor $2 \mathrm{~B}: \mathrm{X}_{2}$ \\
\hline 1 & -1 & 1 \\
\hline 2 & 0 & 1.41 \\
\hline 3 & 0 & 0 \\
\hline 4 & 1 & 1 \\
\hline 5 & -1.41 & 0 \\
\hline 6 & 0 & 0 \\
\hline 7 & 1.41 & 0 \\
\hline 8 & 0 & 0 \\
\hline 9 & 1 & -1 \\
\hline 10 & 0 & 0 \\
\hline 11 & 0 & 0 \\
\hline 12 & -1 & -1 \\
\hline 13 & 0 & -1.41 \\
\hline
\end{tabular}

\section{In Vitro Release}

The dissolution of the multiple components was determined using the basket method. The tests were started with $750 \mathrm{~mL}$ of $0.1 \mathrm{M}$ hydrochloric acid $(\mathrm{HCl})$, and the $\mathrm{pH}$ of the solution was adjusted to 6.8 by adding 250 $\mathrm{mL}$ of a $0.2 \mathrm{~mol} \cdot \mathrm{L}^{-1}$ sodium phosphate liquor after $2 \mathrm{~h}$. The dissolution behavior of PNS from the coated drop pills was measured using a PharmaTest tester (ZRS-8G, TDTF Technology Co., Ltd., China) at a rotating speed of 100 rpm at a stable temperature $\left(37 \pm 0.5^{\circ} \mathrm{C}\right.$.). Based on the transit time of food in the gastrointestinal tract, a series of $5-\mathrm{mL}$ samples were removed at predetermined time points $(1,2,3,4,6,8,10$, and $12 \mathrm{~h})$, filtered through a microporous filter membrane $(0.45-\mu \mathrm{m})$, and $5.0-\mathrm{mL}$ of the medium maintained at $37 \pm 0.5{ }^{\circ} \mathrm{C}$ was added. The released PNS was determined using a high-performance liquid chromatography method.

\section{Pharmacokinetics in Rabbits}

The bioavailability of the pulsatile controlled-release pellets was compared with that of the commercial shuxiong capsule as the reference by using a rabbit model. The animal studies were performed in accordance with the Guide for Care and Use of Laboratory Animals, and the experimental protocol was approved by the Ethics Committee of Guangdong Pharmaceutical University. Twelve healthy rabbits (weight range: $2.5-3.0 \mathrm{~kg}$ ) were divided into two groups for a double cross-over design. One group was orally administered the test preparation ( $0.5 \mathrm{~g}$ of the pulsatile controlled-release pellets per rabbit, each containing $10 \mathrm{mg}$ notoginseng triterpenes) while the other group was administered the reference preparation (commercial conventional capsules containing $10 \mathrm{mg}$ notoginseng triterpenes) via the same route. The rabbits were fasted overnight and allowed free access to water prior to the treatments. Blood samples (approximately $2 \mathrm{~mL}$ ) were collected from the superficial vein of the forelimbs before test drug administration and at predetermined time points afterward $(0,1,2,4,8,12$, and $24 \mathrm{~h})$. Plasma samples were obtained after centrifugation (6500 rpm, $15 \mathrm{~min}$ ) of the blood sample and subsequently stored in a refrigerator at $-20^{\circ} \mathrm{C}$ until the analysis.

\section{Statistical Analysis}

The statistically analyzed data were expressed as mean \pm standard deviation (SD). An analysis of variance (ANOVA) and independent sample $t$-test were performed using the statistical package for the social sciences (SPSS, SAS Institute, Cary, NC, USA) to determine the significant differences between groups, and a p-value $<0.05$ was considered statistically significant.

\section{RESULTS AND DISCUSSION}

\section{Screening of Pulsatile Controlled-Release Pellet Excipients}

The results showed that CCMC-Na or CCMS-Na, which were investigated to determine their suitability as the swelling layer, exhibited typical S-shaped release profiles in line with the pulsatile drug delivery system. 
Furthermore, the swelling property of CCMC-Na and CCMS-Na was more apparent than that of L-HPC or PVPP. L-HPC and PVPP showed no significant pulse release and lag phase, and hence, delayed release could not be achieved. However, the coated drop pills were prone to blockage when CCMS-Na was used as a coating material, thus CCMC-Na was the preferred choice of coating material for the swelling layer in this study.

The individual release profiles for different swelling layer coating weights are shown in Figure 1. Varying the coating weight of the swelling layer had different effects on the release rate of PNS. The data demonstrated that the PNS release profile was similar to that of sustained release formulations of CCMC-Na at a $10 \%$ coating thickness, which may have resulted in the lower coating weight and the insufficient expansion force. Moreover, the accumulated release percentage at $8 \mathrm{~h}$ was greater than $80 \%$ after the lag phase of $4 \mathrm{~h}$ with a coating thickness of $15 \%$. However, all lag times were less than $4 \mathrm{~h}$ when the coating film of the pills was $\geq 20 \%$. Furthermore, the data presented in Figure 2 show that the lag phases were shortened and the PNS release time increased with increasing coating weight. Therefore, the coating weight of $15 \%$ was chosen in this study.

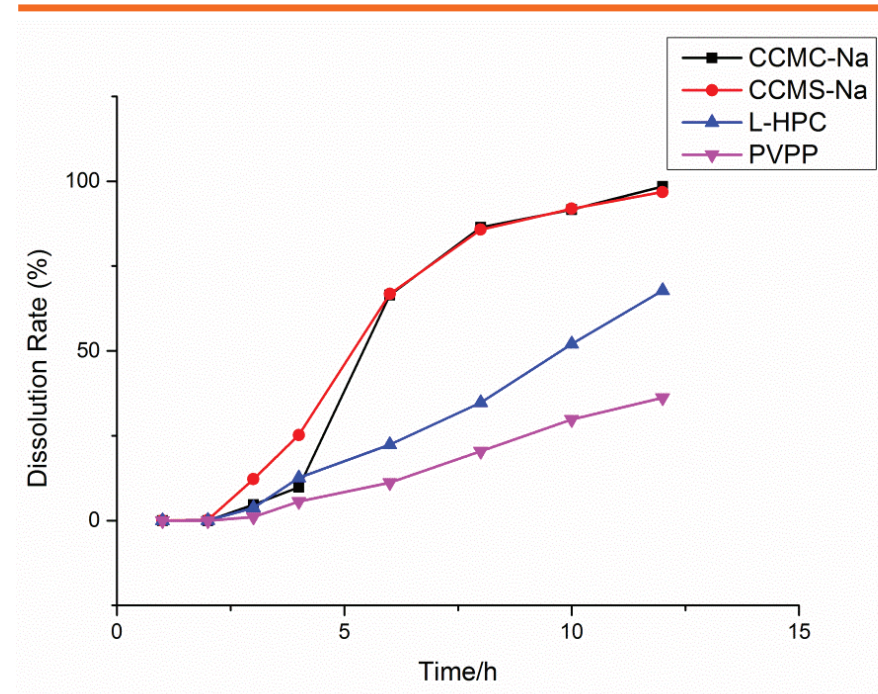

Figure 1. Effect of swelling coating material on release of Panax notoginseng saponin (PNS). CCMC-Na, croscarmellose sodium; CCMS-Na, carboxymethylcellulose sodium; L-HPC, low-substituted hydroxypropyl cellulose; PVPP, polyvinylpolypyrrolidone.

The effect of drug release and lag time on the varied controlled-release coating thickness indicated that the system did not exhibit a lag phase when we set the swelling layer coating thickness at $15 \%$ and the controlledrelease coating thickness at $5 \%$. We observed that the drug release profile showed a classic S-curve. In addition, the profile showed greater than $80 \%$ drug release in $12 \mathrm{~h}$ after a 4-h time lag when the controlled-release coating thickness was reduced to $7 \%$; therefore, this could facilitate pulsatile release. When the coating thickness was increased to $\geq 30 \%$, the corresponding release profile curve became flat with no obvious positioning release and an incomplete drug release. Figure 3 illustrates that the lag phase was extended when the coating thickness increased; however, the increased coating had little effect on time lag after a certain level. This effect may be attributable to the inability of the expansion force to support the burst release. Consequently, the suitable controlled-release coating thickness was determined as $7 \%$.

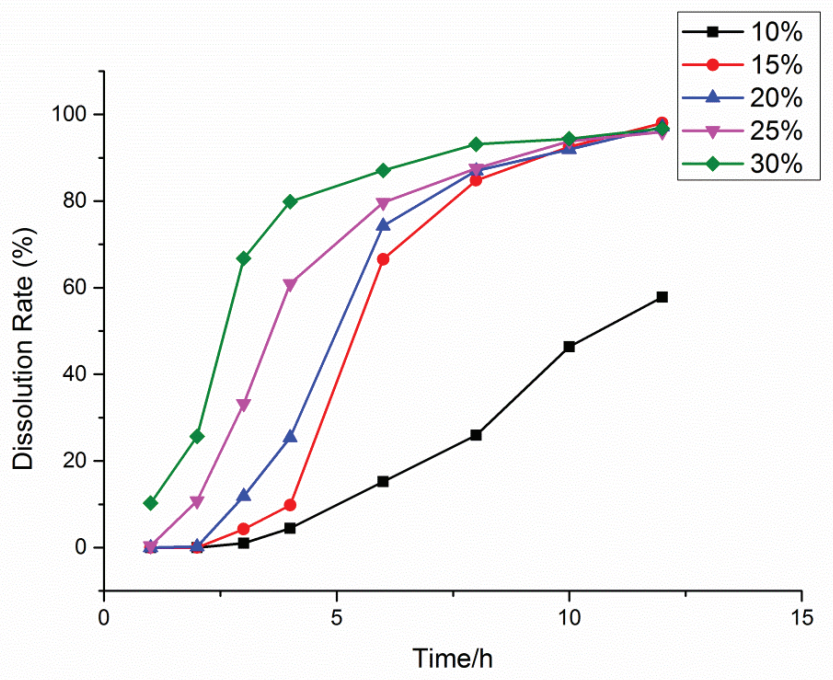

Figure 2. Effect of different swelling layer coating weights on Panax notoginseng saponin (PNS) release.

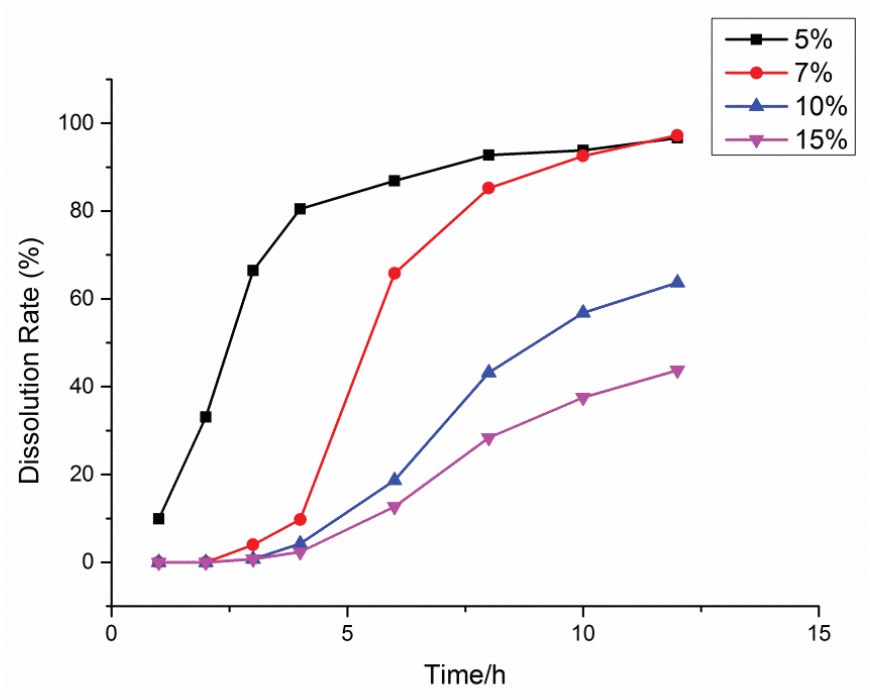

Figure 3. Effect of different controlled-release layer coating weights on release of Panax notoginseng saponins (PNS). 


\section{Model Fitting and Prediction}

The CCD consists of multiple factors and five levels including two-level factorial design points, axial or star points, and center points. According to the preliminary experiments (single factor test results), two selected independent variables (the coating level of swelling layer $\left[\mathrm{X}_{1}: 10-30 \%\right]$ and controlled-release coating layer $\left.\left[X_{2}: 5-15 \%\right]\right)$ were studied at five different levels coded as $( \pm \alpha), \pm 1,0$. The value for alpha (1.414) was intended to fulfill the rotatability of the design. The coded and actual values of the variables are shown in Table 1. The response surface methodology was developed to acquire the optimal preparation conditions by describing the relationships between the variables and the responses. This was achieved using model fitting for the response and the factors, and the established mathematical model was used to describe the three-dimensional response surface. The results are shown in Figure 4.

To predict the most suitable formulation, a quadratic model or higher order polynomial was used to assess the linearity due to the nonlinear relationship between the response and factors. Therefore, the CCD-response surface methodology was suitable for fitting the nonlinear optimization method. The release data were analyzed using the Design-Expert software (version 8.0, Stat-Ease Inc., Minneapolis, MN, USA), and the mathematical model for each response was generated. The results showed that the trinomial model was optimal for this study, which confirmed that the mathematical model has excellent predictability. This observation was based on the predictive analysis, i.e., the model with the highest reliability. The results of the trinomial linear regression analysis $\left(R^{2}\right)$ of each response variable derived by the best fit method are as follows.

$$
\begin{aligned}
& Q_{4 \mathrm{~h}}=6.73+26.58 \times X_{1}-19.81 \times X_{2}-3.32 \times X_{1} \times X_{2}+14.14 \times X_{1}{ }^{2} \\
&+9.69 \times X_{2}^{2}+2.90 \times X_{1}^{2} \times X_{2}-9.67 \times X_{1} \times X_{2}^{2} \\
&\left(R^{2}=0.9558, p=0.0041\right) \\
& Q_{8 \mathrm{~h}}=59.29+23.62 \times X_{1}-14.42 \times X_{2}+7.22 \times X_{1} \times X_{2}-0.11 \times X_{1}^{2} \\
&+3.85 \times X_{2}^{2}-6.67 \times X_{1}^{2} \times X_{2}-17.52 \times X_{1} \times X_{2}^{2} \\
&\left(R^{2}=0.9931, p<0.0001\right)
\end{aligned}
$$$$
Q_{12 \mathrm{~h}}=85.49+13.63 \times X_{1}-6.71 \times X_{2}+13.55 \times X_{1} \times X_{2}-4.55
$$$$
\times X_{1}^{2}-0.15 \times X_{2}^{2}-6.14 \times X_{1}^{2} \times X_{2}-5.78 \times X_{1} \times X_{2}^{2}
$$$$
\left(R^{2}=0.9943, p<0.0001\right)
$$

$Q_{4 \mathrm{~h}}, Q_{8 \mathrm{~h}}$, and $Q_{12 \mathrm{~h}}$ represent the cumulative release of the coated drop pills at 4,8 , and $12 \mathrm{~h}$, respectively. $X_{1}$ and $X_{2}$ represent the coating thickness of the CCMC-Na of the swelling layer (10-30\%) and the coating thickness of the surelease of the controlled release layer (5-15\%).
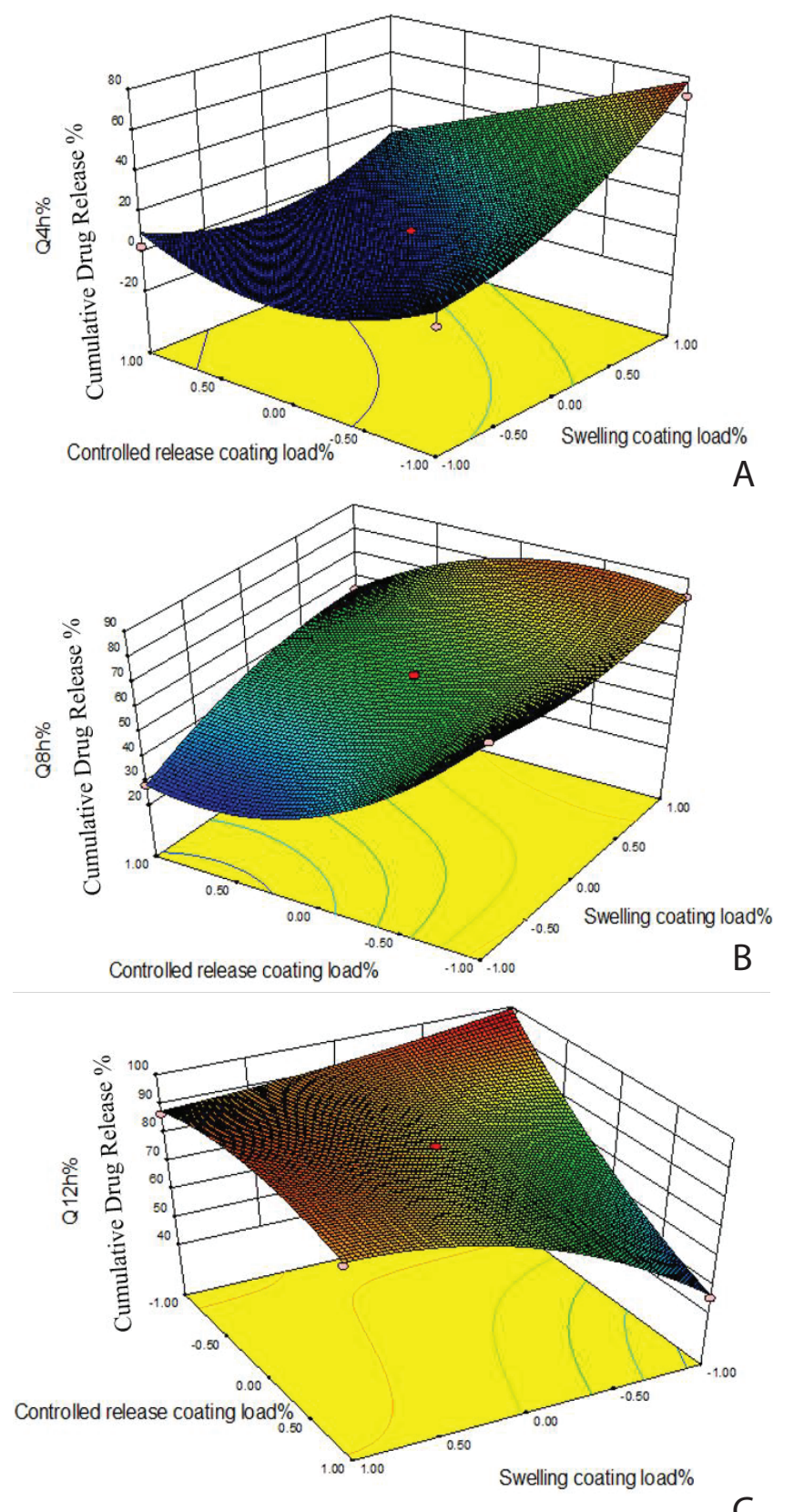

C

Figure 4. Effect of thickness of swelling and controlled release layer coating on cumulative percent drug release at $4(A), 8(B)$, and $12 \mathrm{~h}(C)$.

The prescription was optimized by the curve obtained with swelling and controlled-release coating thickness of $14.27 \%$ and $8.73 \%$, respectively. The results were obtained by extrapolation, which was consistent with the optimal coating formulation of the single factor 
investigation. Thus, the optimum formulation of the coating material used for the swellable coating was CCMC-Na, the controlled-release layer was surelease, and the coating thickness was $15 \%$ and $7 \%$, respectively.

\section{Capability Verification of Model Prediction}

Three batches of the pulsatile controlled-release pellets were prepared according to the optimized preparation process, and their release rates were determined. The results showed that the formulation and preparation process of three batches of coated drop pills were reproducible. The average time lag was $4 \mathrm{~h}$, the cumulative release percentage was greater than $80 \%$, and complete release occurred in $12 \mathrm{~h}$.

\section{Pharmacokinetic Study}

The The delayed release time was approximately $4 \mathrm{~h}$ for the in vitro analysis of pulsatile controlled-release pellets; however, the delayed release time in rabbits in vivo was $40 \mathrm{~min}$. It was difficult to achieve a prolonged delayed release, and the rabbits were used mainly to investigate the performance of the delayed and pulse release in vivo. It is unlikely that the release characteristics of animals and humans are identical, and therefore, the results of this research only provide reference data for human studies. The results of the determination of serum concentration using DAS 3.0 (Mathematical Pharmacology Professional Committee, China) for model fitting showed that the serum drug concentration-time curves were fitted to a two-compartment model, and the pharmacokinetic parameters are shown in Table 3 . The peak plasma concentration $\left(C_{\max }\right)$ increased and the time to reach the $C_{\max }\left(T_{\max }\right)$ of the controlled-release pills was delayed, while the elimination half-time $\left(t_{1 / 2}\right)$ was also prolonged compared to that of the conventional pills, due to the delayed effect. The area under the curve from time 0 to infinity $\left(\mathrm{AUC}_{0-\infty}\right)$ of the delayed-release pulsatile pills was $242.682 \mathrm{mg} / \mathrm{L} \times \mathrm{h}$ compared to $75.439 \mathrm{mg} / \mathrm{L} \times \mathrm{h}$ for the conventional pills. This result shows that the value of the pulsatile pills was approximately 3.2 times that of the conventional pills, indicating that the pulsatile controlledrelease pill had good bioavailability. The mean residence time (MRT) of the pulsatile pills was calculated using the statistical distance (non-compartment model), and the value (15.204 h) was longer than that of the conventional formulation (4.693 h) at $10.511 \mathrm{~h}$. These results showed that the pulsatile controlled-release pills exhibited a significant delay in the release and there was a correlation between the in vivo and in vitro release process compared to the conventional preparation.
Table 3. Pharmacokinetic Parameters of a Single Dose of Coated Drop Pills

\begin{tabular}{|c|c|c|}
\hline Parameters & Drop pills & Film-Controlled Drop Pills \\
\hline$\alpha$ & 0.7 & 0.173 \\
\hline$\beta$ & 0.468 & 0.122 \\
\hline$\kappa$ & 305.866 & 287.974 \\
\hline Ka & 1.111 & 0.364 \\
\hline$t_{1 / 2}(\mathrm{~h})$ & 0.991 & 4.003 \\
\hline$K 10(1 / \mathrm{h})$ & 5.356 & 0.14 \\
\hline$A \cup C_{(0-\infty)}(\mathrm{mg} / \mathrm{L} \times \mathrm{h})$ & 75.439 & 242.682 \\
\hline$T_{\max }(\mathrm{h})$ & 5 & 8 \\
\hline$C_{\max }(\mathrm{mg} / \mathrm{L})$ & 17.905 & 22.518 \\
\hline MRT (h) & 4.693 & 15.204 \\
\hline
\end{tabular}

\section{CONCLUSION}

A novel time- and controlled-release coating formulation was developed by investigating the factors affecting the coating of the test prescription. The results of the in vitro simulation of the physiological conditions of the human gastrointestinal tract show that the sustained-release dripping pill exhibited time-dependent gradient-release characteristics. Moreover, the results indicate that the pulsatile release of a coated drop pill has gradient pulsatile characteristics in the in vivo study in rabbits. This effect was equivalent to that observed following multi-dose administration and achieved a sustained release effect.

\section{CONFLICT OF INTEREST}

The authors disclosed no conflicts of interest related to this study.

\section{REFERENCES}

1. Efferth,T.; Koch, E. Complexinteractions between phytochemicals. The multi-target therapeutic concept of phytotherapy. Curr. Drug Targets 2011, 12, 122-132.

2. Kannaiyan, R.; Shanmugam, M. K.; Sethi, G. Molecular targets of celastrol derived from Thunder of God Vine: potential role in the treatment of inflammatory disorders and cancer. Cancer Lett. 2011, 303, 9-20. DOI: 10.1016/j.canlet.2010.10.025.

3. Kitano, H. A robustness-based approach to systems-oriented drug design. Nat. Rev. Drug Discov. 2007, 6, 202-210. DOI: $10.1038 / \mathrm{nrd} 2195$.

4. Wang, J.; van der Heijden, R.; Spruit, S.; Hankermeier, T.; Chan, K.; van der Greef, J.; Xu, G.; Wang, M. Quality and safety of Chinese herbal medicines guided by a systems biology perspective. J.Ethnopharmacol.2009, 26, 31-41. DOI: 10.1016/j. jep.2009.07.040. 
5. Yuan, R.; Lin, Y. Traditional Chinese medicine: an approach to scientific proof and clinical validation. Pharmacol. Ther. 2000, 86, 191-198. DOI: 10.1016/S0163-7258(00)00039-5.

6. Wang, X.; Sun, Y.; Sun, K.; Ding, Y.; Yuan, R. Review: separation and pharmacology of chiral compounds in traditional Chinese medicine. Anal. Lett. 2017, 50, 33-49. DOI: 10.1080/00032719.2016.1169540.

7. Xing, S.; Wang, M.; Peng, Y.; Li, X. Effects of intestinal microecology on metabolism and pharmacokinetics of oral Wogonoside and Baicalin. Nat. Prod. Commun. 2017, 12, 509-514.

8. Liu, S.; Liang, Y. Z.; Liu, H. T. Chemometrics applied to quality control and metabolomics for traditional Chinese medicines. J. Chromatogr. B 2016, 1015-1016, 82-91. DOI: 10.1016/j. jchromb.2016.02.011.

9. Li-Weber, M. New therapeutic aspects of flavones: the anticancer properties of Scutellaria and its main active constituents Wogonin, Baicalein and Baicalin. Cancer Treat. Rev. 2009, 35, 57-68. DOI: 10.1016/j.ctrv.2008.09.005.

10. Shi, J.; Cao, B.; Wang, X. W.; Aa, J. Y.; Duan, J. A.; Zhu, X. X.; Wang, G. J.; Liu, C. X. Metabolomics and its application to the evaluation of the efficacy and toxicity of traditional Chinese herb medicines. J. Chromatogr. B 2016, 1026, 204-216. DOI: 10.1016/j. jchromb.2015.10.014.

11. Zhao, Z.; Liu, X.; Wang, Y.; Liu, G.; Zhang, Z.; Zhao, Z.; Cheng, H. In vitro antioxidative and immunological activities of polysaccharides from Zizyphus Jujuba cv. Muzao. Int. J. Biol. Macromol. 2017, 95, 1119-1125. DOI: 10.1016/j.jjbiomac.2016.10.102.

12. Lv, Y.; Fu, J.; Shi, X.; Yang, Z.; Han, S. Screening allergic components of Yejuhua injection using LAD2 cell membrane chromatography model online with high performance liquid chromatography-ion trap-time of flight-mass spectrum system. J. Chromatogr. B 2017, 1055-1056, 119-124. DOI: 10.1016/j.jchromb.2017.04.045.

13. Xie, Y.; Yi, Y.; Hu, X.; Shangguan, M.; Wang, L.; Lu, Y.; Qi, J.; Wu, $W$. Synchronous microencapsulation of multiple components in silymarin into PLGA nanoparticles by an emulsification/solvent evaporation method. Pharm. Dev. Technol. 2016, 21, 672-679. DOI: 10.3109/10837450.2015.1045616.

14. Song, H. T.; Zhang, Q.; Kong, L. L.; Chen, D. W.; He, Z. G. [Preparation of Shuxiong micropellets by centrifugal granulation technology].
[In English] Zhongguo Zhong Yao Za Zhi 2006, 31, 1147-1150.

15. Song, H. T.; Zhang, Q.; Wang, H. J.; Guo, X. J.; Chen, D. W.; He, Z. G. Preparation of the traditional Chinese medicine compound recipe Shuxiong sustained-release capsules by multiparticulate time-controlled explosion technology. Die Pharmazie Int. J. Pharm. Sci. 2007, 62, 372-377. DOI: 10.1691/ph.2007.5.6097.

16. Yao, C.; Yang, W.; Si, W.; Pan, H.; Qiu, S.; Wu, J.; Shi, X.; Feng, R., Wu, W.; Guo, D. A strategy for establishment of practical identification methods for Chinese patent medicine from systematic multicomponent characterization to selective ion monitoring of chemical markers: Shuxiong tablet as a case study. RSC Adv 2016, 6, 65055-65066. DOI: 10.1039/C6RA10883K.

17. Lian, F.; Wu, L.; Tian, J.; Jin, M.; Zhou, S.; Zhao, M.; Wei, L.; Zheng, Y.; Wang, Y.; Zhang, M.; Qin, W.; Wu, Z.; Yuan, C. S.; Tong, X. The effectiveness and safety of a danshen-containing Chinese herbal medicine for diabetic retinopathy: a randomized, double-blind, placebo-controlled multicenter clinical trial. J. Ethnopharmacol. 2015, 164, 71-77. DOI: 10.1016/j.jep.2015.01.048.

18. Zou, P. Traditional Chinese medicine, food therapy, and hypertension control: a narrative review of Chinese literature. Am. J. Chin. Med. 2016, 44, 1579-1594. DOI: 10.1142/ S0192415X16500889.

19. Kim, D. H. Chemical diversity of Panax ginseng, Panax quinquifolium, and Panax notoginseng. J. Ginseng Res. 2012, 36, 1-15. DOI: 10.5142/jgr.2012.36.1.1.

20. Lee, C. H.; Kim, J. H. A review on the medicinal potentials of ginseng and ginsenosides on cardiovascular diseases. J. Ginseng Res. 2014, 38, 161-166. DOI: 10.1016/j.jgr.2014.03.001.

21. Liu, J.; Wang, Y.; Qiu, L.; Yu, Y.; Wang, C. Saponins of Panax notoginseng: chemistry, cellular targets and therapeutic opportunities in cardiovascular diseases. Expert Opin. Investig. Drugs 2014, 23, 523-539. DOI: 10.1517/13543784.2014.892582.

22. Wang, T.; Guo, R.; Zhou, G.; Zhou, X.; Kou, Z.; Sui, F.; Li, C.; Tang, L.; Wang, Z. Traditional uses, botany, phytochemistry, pharmacology and toxicology of Panax notoginseng (Burk.) F.H. Chen: A review. J. Ethnopharmacol. 2016, 188, 234-258. DOI: 10.1016/j. jep.2016.05.005. 